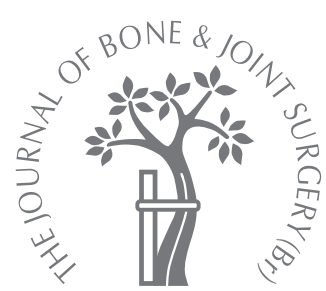

F. von Knoch, U. Reckord, M. von Knoch, C. Sommer

From the Kantonsspital Graubünden, Chur, Switzerland

\title{
Fracture of the lateral process of the talus in snowboarders
}

\begin{abstract}
We investigated the clinical and radiological outcome after unilateral fracture of the lateral process of the talus in 23 snowboarders with a mean follow-up of 3.5 years (12 to 76 months). In this consecutive cohort study both operative and non-operative cases were considered.

The mean American Orthopaedic Foot and Ankle Society hindfoot score was 94 (82 to $100)$. The non-operative group of seven with a minimally-displaced fracture scored higher (98 points) than the operative group of 16 with displaced or unstable fractures (93 points). In $\mathbf{8 8 \%}$ of operative cases, significant concomitant hindfoot injuries were found at operation. All but eight (35\%) patients (six operative and two non-operative) regained their pre-injury level of sporting activity. Subtalar osteoarthritis was present in nine $(45 \%)$ of the 20 patients available for radiological review, including one late-diagnosed non-operative case and eight operative cases with associated injuries or fracture comminution.

The outcome after fracture of the lateral process of the talus in snowboarders is favourable provided an early diagnosis is made and adequate treatment, which is related to the degree of displacement and associated injuries, is undertaken.
\end{abstract}

The lateral process of the body of the talus is a broad-based wedge-shaped prominence that articulates dorsolaterally with the fibula and inferomedially with the os calcis, thereby forming the lateral part of the subtalar joint. ${ }^{1}$ Fracture of the lateral process is seen typically in snowboarders. Before the development of this sport, these fractures were rare and generally associated with motor vehicle accidents, falls from a height, or simple inversion injuries. ${ }^{1,2}$ With the increasing popularity of snowboarding, fractures of the lateral process of the talus occur more frequently and account for $2.3 \%$ of snowboarding injuries. ${ }^{3}$

The mechanism of injury is believed to be dorsiflexion of the ankle with inversion of the foot. ${ }^{4}$ Recent evidence from in vitro biomechanical $^{5}$ and clinical $^{6}$ studies suggests that dorsiflexion, axial impaction, eversion and external rotation are involved. According to Hawkins, ${ }^{1}$ these fractures may be classified as non-articular (type I), a single fragment involving the talofibular articulation and subtalar joint (II), and comminuted fractures, also involving both articulations (III).

Fracture of the lateral process of the talus has often been misdiagnosed as a lateral ankle sprain., ${ }^{3,7}$ The sequelae of missed fractures, particularly types II and III, include malunion, nonunion, long-term disability and degenerative arthritis of the subtalar joint. ${ }^{3}$ The diagnosis can usually be established by a careful history, clinical examination and standard plain radiographs. In selected cases, CT may be needed to confirm a suspected fracture. $^{8}$

The clinical outcome of fractures of the lateral process of the talus has hitherto been limited to case reports ${ }^{8,9}$ and one formal series (20 cases) of clinical and radiological results in snowboarders. ${ }^{6}$ We are not aware of any study that provides detailed information on associated injuries and outcome following fractures of the lateral process of the talus sustained while snowboarding.

\section{Patients and Methods}

Between 1995 and 2001, 26 consecutive snowboarders (23 men, three women) sustained a unilateral fracture of the lateral process of the talus. All were treated at a level-one trauma centre, where the diagnosis was established immediately in all but three patients. One was undiagnosed for five months and two were referred with a delayed diagnosis. The criteria for suspicion of a lateral process fracture include a history of ankle sprain during snowboarding, and localised pain and tenderness $1 \mathrm{~cm}$ below 


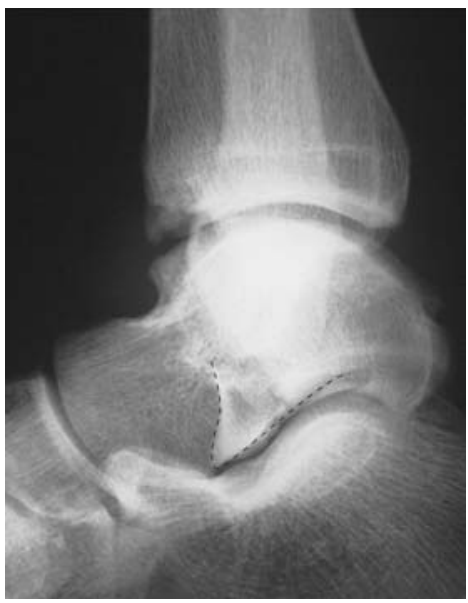

Fig. 1a

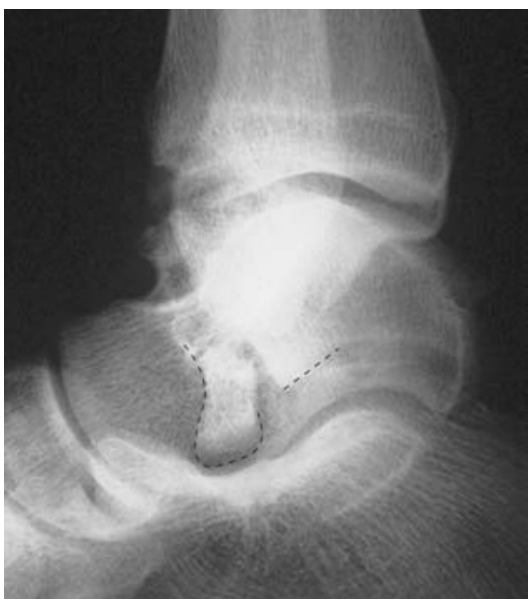

Fig. 1b

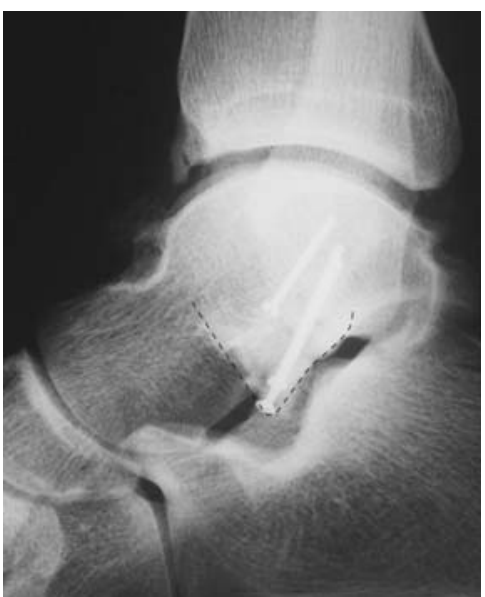

Fig. 1c

Lateral radiographs of a 23-year-old male snowboarder's right ankle showing a single large-fragment type II fracture of the lateral process of the talus, a) immediately after injury, b) five months later and c) after surgery. The fracture was initially misdiagnosed as minimally displaced despite a crooked $\checkmark$ shape (dotted line) of the lateral process (a). This is a positive $V$ sign. There was malunion after conservative treatment (b). Following surgery (corrective osteotomy) eight months after injury, the $\mathrm{V}$ sign is negative (c).

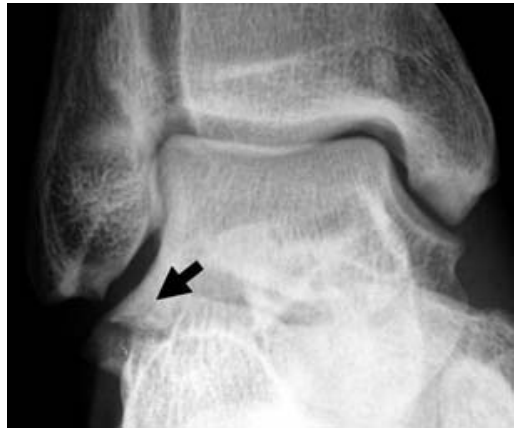

Fig. 2a

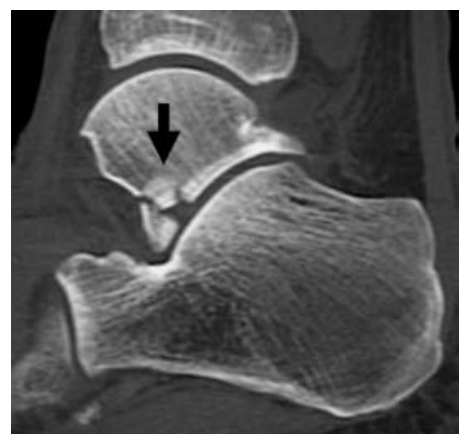

Fig. $2 d$

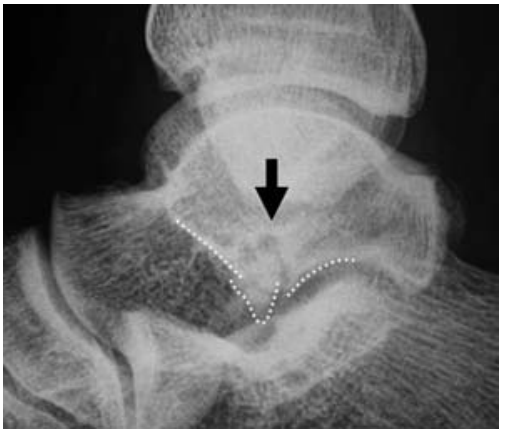

Fig. $2 b$

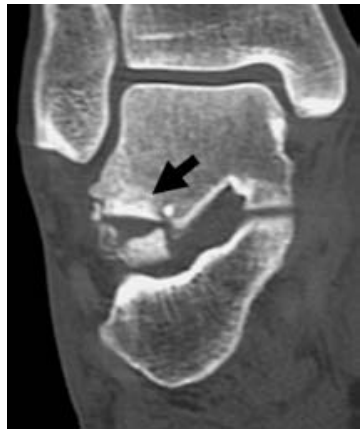

Fig. 2c

a) Anteroposterior and b) lateral radiographs, and c) coronal and d) sagittal CT scans of a 22year-old male snowboarder's right ankle. There is a displaced multifragmentary type III fracture of the lateral process of the talus (arrow). Note a positive $V$ sign (b).

the tip of the lateral malleolus. ${ }^{4}$ Demographic, clinical and radiological data including anteroposterior (AP) and lateral views of the ankles were recorded. In the latter, an intact lateral process of the talus has a symmetrical V-shaped contour. With a displaced fracture, the V shape is crooked or otherwise asymmetrical (Fig. 1). We designated this appear- ance a positive $\mathrm{V}$ sign. In operative cases we undertook Broden's view (AP radiograph in a $15^{\circ}$ caudocranial direction with $45^{\circ}$ to $60^{\circ}$ of internal rotation of the foot) and a 'reversed Broden's view' (AP view in a $10^{\circ}$ caudocranial direction with $30^{\circ}$ to $45^{\circ}$ of external rotation of the foot) during the operation. Broden's view highlights the postero- 


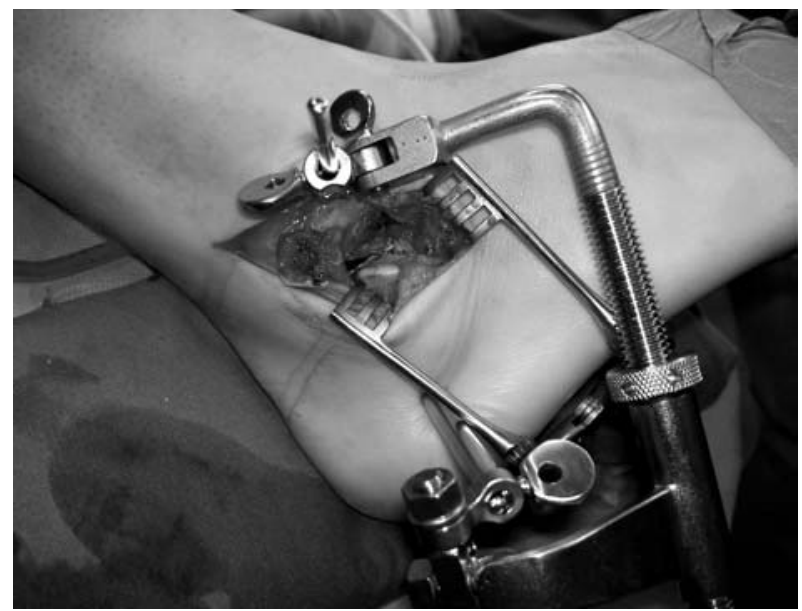

Fig. 3a

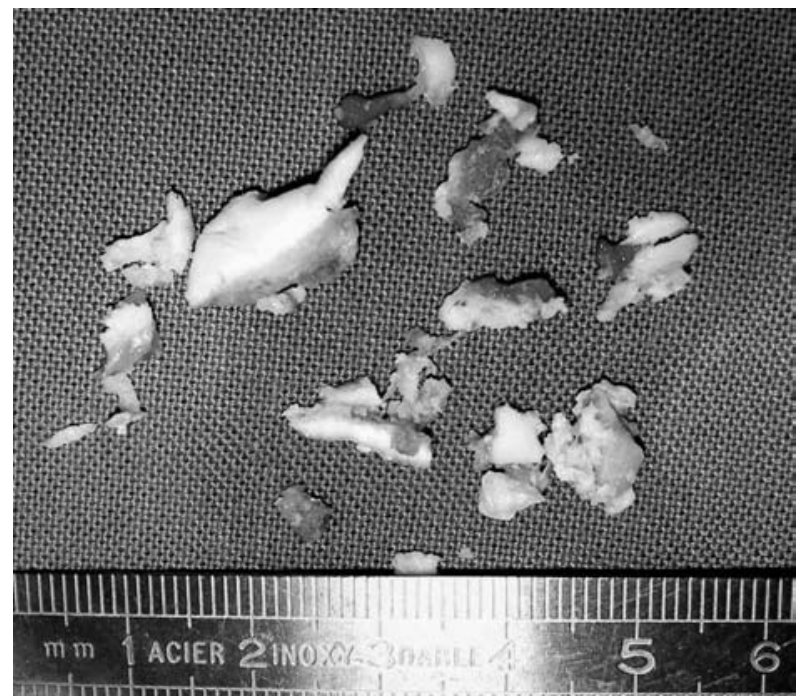

Fig. 3c

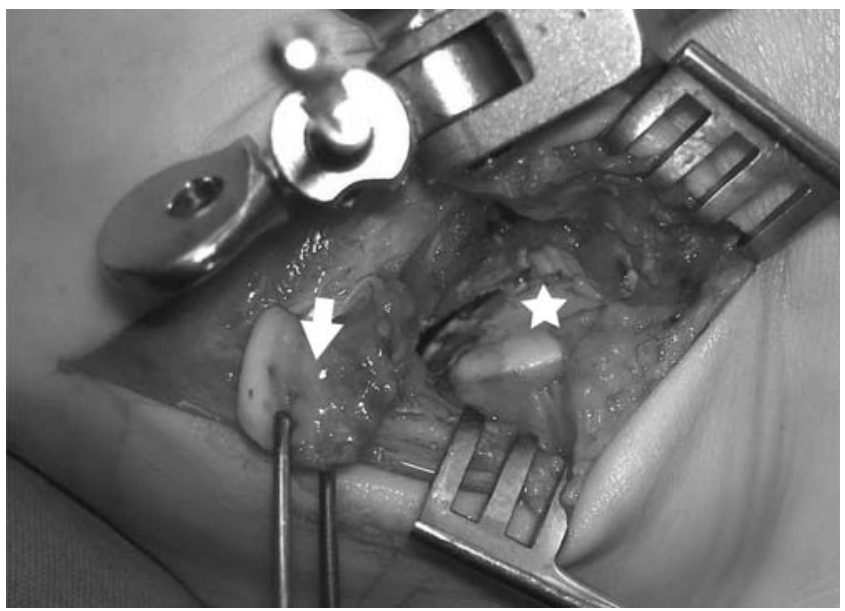

Fig. $3 b$

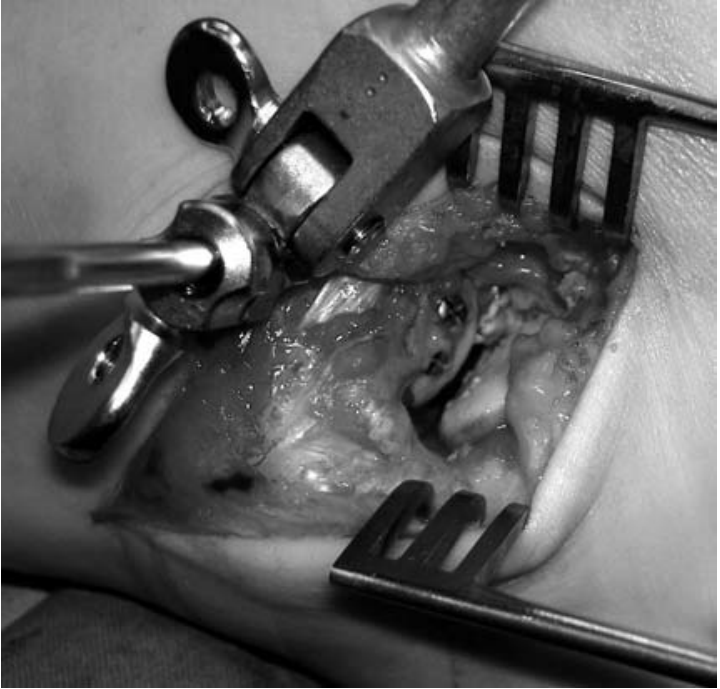

Fig. 3d

Intra-operative pictures of the same patient as in Figure 2. A small AO distractor is applied. a) Overview and b) close-up views before open reduction and internal fixation show a large fragment (arrow) and subtalar calcaneal articular damage (star). c) Multiple loose osteochondral fragments were removed and d) anatomical reduction was achieved using two $2.0 \mathrm{~mm}$ lag screws.

lateral aspect of the lateral process. The reversed Broden's view exposes the anterolateral aspect of the fracture gap and outlines small fracture gaps or articular step-offs. Usually, CT scans (Fig. 2) were done for better determination of fracture type. ${ }^{1}$ A CT scan was specifically indicated to confirm a suspected fracture of the lateral process that was equivocal on conventional radiographs, to assess the displacement of a fracture when treatment was undecided, and to detect suspected associated fractures. Treatment was based on fracture type, the degree of displacement and the presence or absence of associated injuries. Type I and minimally displaced $(<1 \mathrm{~mm})$ type II and III fractures without significant associated injuries were treated non-operatively. The remainder were treated by open reduction and internal fixation.
Of the 26 fractures of the lateral process of the talus, we retrospectively reviewed the clinical and radiological outcome of 23 snowboarders (21 men, two women) with a mean follow-up of 3.5 years (12 to 76 months). The mean age of the patients at the time of injury was 31 years (16 to 43 ). Three patients were lost to follow-up. The fractures were classified as type I (one case); type II (15 cases, 11 operative) and type III (seven cases, five operative). The leading foot was affected in 16 patients $(70 \%)$. The mechanism of injury involved dorsiflexion and axial impaction in all cases with rotation in 15 $(65 \%)$ and hindfoot eversion in two $(9 \%)$. The boots worn were soft in nine patients (39\%), hybrid in three $(13 \%)$ and stiff in ten $(44 \%)$. In one patient the boot style was not recorded. There was no statistically signif- 


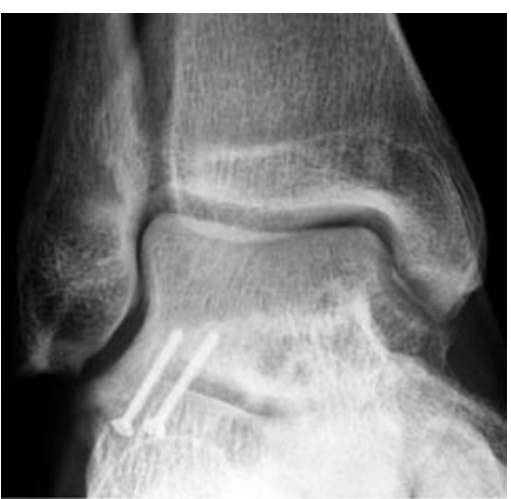

Fig. 4a

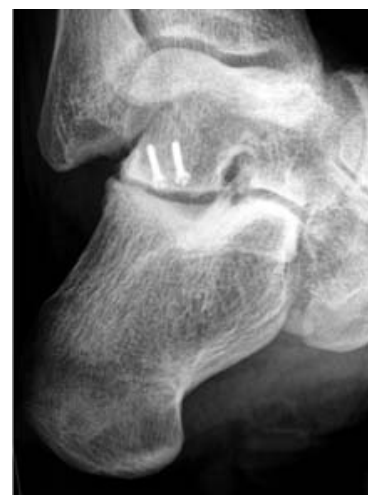

Fig. $4 \mathrm{~b}$

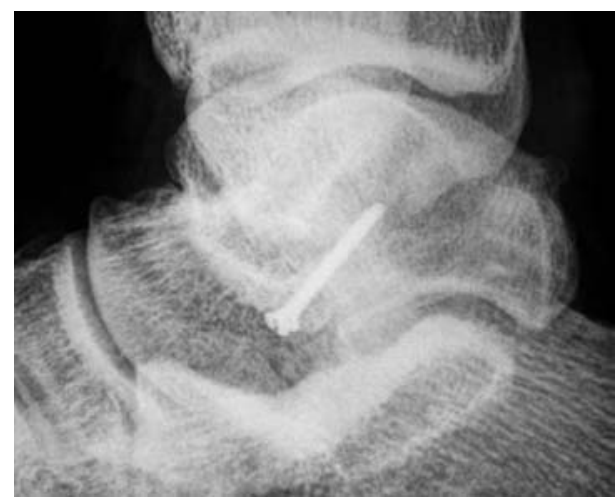

Fig. 4c

Radiographs of the patient in Figures 2 and 3, 18 months after open reduction and internal fixation. a) Anteroposterior, b) Brodens and c) lateral projections show consolidation and adequate reduction of the fracture of the lateral process of the talus and mild degenerative changes of the subtalar joint.

icant association between the type of fracture and the kind of boot ( $\mathrm{p}=0.79$, chi-squared test).

Non-operative treatment. Seven patients $(30 \%)$ were immobilised in an aircast device for six weeks and allowed partial weight-bearing of $15 \mathrm{~kg}$. When fracture healing was confirmed radiologically, full weight-bearing was allowed with graded return to previous activities.

Operative treatment. Of the 23 patients, $16(70 \%)$ underwent open reduction and internal fixation (Fig. 3). The patient was supine with a thigh tourniquet. Through a curved incision from the distal end of the fibula to the anterior process of the calcaneus, the peroneal sheath and anterior talofibular and calcaneofibular ligaments were exposed. Between these ligaments the subtalar joint was opened to expose the lateral process of the talus. In most cases the capsule was already ruptured. The displaced and often stripped fragments were displaced anteriorly and the subtalar joint explored. In some cases a small AO distractor between the talar neck and the calcaneal tubercle allowed better exposure (Figs $3 \mathrm{a}$ and $3 \mathrm{~b}$ ). Cartilaginous flakes and small osteochondral fragments were removed (Fig. 3c) and the main fragment reduced under direct vision (Fig. 3d). In most cases internal fixation of large fragments was achieved by two $2.0 \mathrm{~mm}$ AO titanium lag screws in a mediocranial direction (Fig. 4). For smaller fragments, one screw and a $1.0 \mathrm{~mm}$ Kirschner (K)-wire provided stability. For multifragmentary fractures, a $2.0 \mathrm{~mm}$ AO titanium T-plate was used as a buttress. Where there was cranial impaction cancellous autograft was used for the defect. If the peroneal tendon was subluxed, its sheath was repaired. Ruptured calcaneofibular ligaments were repaired and additional fractures of the talar body and the sustentaculum tali stabilised by screw fixation, through a separate approach if necessary. The joint capsule was sutured and the wound closed. Following bed rest and elevation for two or three days, the patients were mobilised partial-weight-bearing
(10 kg to $15 \mathrm{~kg}$ ) for six weeks. Also, when ligament or tendon sheath repair was necessary, the ankle was immobilised for six weeks in a walking boot. After six weeks, full-weight bearing was allowed.

Clinical evaluation included assessment of tenderness, hindfoot alignment and range of movement of the ankle and subtalar joints using the American Orthopaedic Foot and Ankle Society (AOFAS) hindfoot score. ${ }^{10}$ Subsequent operations, walking aids, the use of analgesia and the time taken to maximal recovery, sporting activities, occupation and overall satisfaction were recorded.

A total of 20 patients had post-operative radiographs including AP, lateral and Broden's view (Fig. 4). These were analysed for fracture reduction, union and the presence of osteoarthritis using a four-grade classification system. ${ }^{6}$

\section{Results}

Of the 16 operative patients, $13(81 \%)$ had their operations immediately (Fig. 2), two (13\%) at 15 days and five months, respectively because of delayed diagnosis, and one $(6 \%)$ at eight months as a result of malunion (Fig. 1). A total of $14(88 \%)$ had significant associated hindfoot injuries on CT scans or at operation, including calcaneal cartilage lesions (11 patients, 69\%), and ligamentous or tendon injuries (ten patients, $63 \%$ ), of which eight $(50 \%)$ were ruptures of the calcaneofibular ligament, five $(31 \%)$ were dislocations of the peroneal tendon because of rupture of its sheath, and one $(6 \%)$ was a rupture of the anterior fibulotalar ligament. Subluxation of the subtalar joint was found in two cases, fracture of the sustentaculum tali in one, and fracture of the trochlear process of the calcaneum in one. There were no post-operative complications. Three patients underwent uneventful removal of their hardware at eight months (two patients) and ten months (one patient) postoperatively. The patient who had surgery five months after injury underwent revision of the peroneal tendon sheath 
Table I. American Orthopaedic Foot and Ankle Society hindfoot scores ${ }^{10}$ (values expressed as mean (range))

\begin{tabular}{lllll}
\hline & Total (maximum 100) & Pain (maximum 40) & Function (maximum 50) & Alignment (maximum 10) \\
\hline All cases $(n=23)$ & $94(82$ to 100$)$ & $36(30$ to 40$)$ & $48(42$ to 50$)$ & $10(10$ to 10$)$ \\
Non-operative $(n=7)$ & $98(87$ to 100$)$ & $37(30$ to 40$)$ & $49(47$ to 50$)$ & $10(10$ to 10$)$ \\
Operative $(n=16)$ & $93(82$ to 100$)$ & $35(30$ to 40$)$ & $48(42$ to 50$)$ & $10(10$ to 10$)$ \\
\hline
\end{tabular}

repair and drilling of an osteonecrotic focus of the calcaneum. No other patient required further surgery.

At the most recent clinical review the mean AOFAS score was 94 (82 to 100; Table I). The non-operative group with minimally-displaced fractures scored higher (98 points) than did the operative group (93 points). All patients had normal ankle movement. Hindfoot movement was normal or mildly restricted $(<25 \%)$ in all but one patient in the operative group who had moderate restriction. No patient required walking aids and only one patient from the operative group had changed occupation because of their injury. All but two non-operative and six operative patients who had significant associated injuries, including calcaneal cartilage lesions (three patients), ruptures of the calcaneofibular ligament (four patients), dislocations of the peroneal tendon (two patients), subluxation of the subtalar joint (two patients), fracture of the sustentaculum tali (one patient), and fracture of the trochlear process of the calcaneum (one patient) regained the same level of sporting activity as before injury. No patient used medication for pain, except one who occasionally took non-steroidal antiinflammatories. The mean time to achieve maximum function was six months (2 to 12 ). Patients with minimally-displaced fractures recovered twice as fast (mean 13 weeks) as the operative group (mean 27 weeks). The majority of patients $(87 \%)$ were satisfied with their treatment and perceived that they had recovered to their pre-injury level. A total of 18 hindfeet $(78 \%)$ were rated by the patient as excellent; two good and one fair (non-operative patient with delayed diagnosis). Two reported a poor outcome, one operative patient with a large calcaneal chondral lesion and subsequent subtalar osteoarthritis, and one non-operative patient, for no obvious reason.

At latest radiological follow-up all fractures of the lateral process of the talus appeared healed. Reduction was adequate in all but the non-operative case where the patient was initially misdiagnosed and later referred. Subtalar osteoarthritis was present in nine patients (nine of 20; $45 \%$ ) including one non-operative case referred with delayed diagnosis and eight surgical cases complicated by associated injuries or comminution.

\section{Discussion}

A high degree of suspicion is warranted in snowboarders presenting with anterolateral ankle pain. The diagnosis of fracture of the lateral process of the talus can usually be made by a careful history, clinical examination and standard radiographs. In our experience, the positive $\mathrm{V}$ sign is pathognomic for displaced fractures. Additional CT scan- ning is indicated for a suspected fracture, or to provide additional information on degree of displacement or associated fractures. 3,7

There are few data on associated injuries in patients who sustain this fracture while snowboarding. In our study, the majority $(88 \%)$ of patients who underwent operative treatment had significant concomitant hindfoot injuries, mostly cartilaginous or ligamentous. Fractures of the lateral process were rarely associated with other fractures. One patient had an associated fracture of the sustentaculum tali and one a fracture of the trochlear process of the calcaneum. In the non-operative group, soft-tissue injuries such as dislocation of the peroneal tendon were excluded clinically. The use of MR imaging, although potentially useful in identifying concomitant injuries such as subtalar chondral damage, was not undertaken as part of our acute diagnostic work-up. Minimally displaced fractures of the lateral process represent a less severe, low-energy injury accompanied by fewer and lesser associated injuries and may be treated conservatively with a good outcome.

Our patients were permitted only to bear a restricted weight of $15 \mathrm{~kg}$ for six weeks, as most fractures of the lateral process (types II and III) involve the subtalar joint. We believe that bearing full weight with some degree of hindfoot eversion during the first weeks after trauma or open reduction and internal fixation, may subject the fragments to axial and shearing forces that could compromise reduction, healing and outcome.

Our regimen gave satisfactory results. The majority of patients were satisfied and had high AOFAS scores. These encouraging results are supported by Valderrabano et al, ${ }^{6}$ who found significantly higher AOFAS scores after operative treatment. We do not know why their operative patients scored higher. In our cohort, all patients achieved high AOFAS scores, but non-operative cases scored slightly better. The operative cases included multifragmentary and displaced fractures as a result of high-energy trauma. Despite the greater severity of injury, the differences in outcome between the groups were minor. However, in contrast to these promising clinical results, the presence of mild or moderate degenerative changes in $45 \%$ of the subtalar joints is concerning. Degenerative changes were found mostly in surgically-treated patients with displaced fractures and significant associated injuries, the foremost being subtalar chondral lesions. However, in this retrospective study, the extent of chondral lesions was not recorded in all cases, preventing study of the relationship between chondral lesions and outcomes. 
In conclusion, this study demonstrates that non-operative treatment is successful for undisplaced fractures of the lateral process of the talus in the absence of associated injuries. Operative intervention with open reduction and internal fixation is successful for displaced fractures or when there are additional injuries. The outcome in snowboarders is favourable, provided the diagnosis is made early and adequate treatment is undertaken.

No benefits in any form have been received or will be received from a commercial party related directly or indirectly to the subject of this article.

\section{References}

1. Hawkins LG. Fracture of the lateral process of the talus. J Bone Joint Surg [Am] 1965;47-A:1170-5

2. Mukherjee SK, Pringle RM, Baxter AD. Fracture of the lateral process of the talus: a report of thirteen cases. J Bone Joint Surg [Br] 1974;56-B:263-73.
3. Kirkpatrick DP, Hunter RE, Janes PC, Mastrangelo J, Nicholas RA. The snowboarder's foot and ankle. Am J Sports Med 1998;26:271-7.

4. McCrory P, Bladin C. Fractures of the lateral process of the talus: a clinical review. Clin J Sport Med 1996;6:124-8.

5. Funk JR, Srinivasan SC, Crandall JR. Snowboarder's talus fractures experimentally produced by eversion and dorsiflexion. Am J Sports Med 2003;31:921-8.

6. Valderrabano V, Perren T, Ryf C, Rillmann P, Hintermann B. Snowboarder's talus fracture: treatment outcome of 20 cases after 3.5 years. Am J Sports Med 2005;33:871-80.

7. Noble J, Royle SG. Fracture of the lateral process of the talus: computed tomographic scan diagnosis. Br J Sports Med 1992;26:245-6.

8. Tucker DJ, Feder JM, Boylan JP. Fractures of the lateral process of the talus: two case reports and a comprehensive literature review. Foot Ankle Int 1998;19:641-6.

9. Sharma S. Fracture of lateral process of the talus presenting as ankle pain. Emerg Med J 2003;20:2

10. Kitaoka HB, Alexander IJ, Adelaar RS, et al. Clinical rating systems for the anklehindfoot, midfoot, hallux, and lesser toes. Foot Ankle Int 1994;15:349-53. 